\title{
The overshadowing of background stimuli by an informative CS in aversive Pavlovian conditioning with rats
}

\author{
F. J. ODLING-SMEE \\ Brunel University, Uxbridge, Middlesex, England
}

\begin{abstract}
Two experiments investigated the capacity of a nominal CS to overshadow background stimuli. Rats received CS-shock pairings in one compartment of a double-compartment apparatus. After training, the shock compartment was re-presented but in the absence of both the CS and shock, and overshadowing was assessed by measuring the rats' latency to enter the compartment. In the first experiment, predictive values of temporal cues provided by CS onset and CS offset were manipulated. Rats readily entered the shock compartment, provided shock onset had previously been predicted by CS onset or CS offset. This indicates that background cues in the shock compartment had acquired little associative strength during training. In contrast, rats avoided the shock compartment under every condition in which shock onset had not previously been predicted by either CS onset or CS offset. In the second experiment, a precise temporal cue provided by CS onset was dissociated from other factors arising from CS-shock contiguity. It was found that rats' reactions to the shock compartment after training was governed by both temporal and contiguity factors. The results are relevant to theories which state that conditioning is a function of a competitive relationship between stimuli present in an animal's environment during training.
\end{abstract}

Several theories of learning propose that conditioning is a function of a competition for associative strength between all the stimuli present in an animal's environment to which a conditioned response could potentially become attached (Mackintosh, 1975; McHose \& Moore, 1976; Rescorla \& Wagner, 1972; Sutherland \& Mackintosh, 1971). These theories are primarily concerned with the relationship between components of compound conditioned stimuli (CSs), but because background stimuli are known to control responding in many conditioning situations, the same theories also consider the relationship between background stimuli and nominal CSs.

The purpose of this study was to examine one aspect of the relationship between background stimuli and a nominal CS. If unsignaled unconditioned stimuli (UCSs) are presented in a training environment, then theoretically, associative strength should accrue to background or contextual stimuli. If the UCS remains constant, then the background stimuli should "block" conditioning to any subsequently introduced CS, the blocking being identical to the phenomenon demonstrated by Kamin (1969). Conversely, if CS-UCS pairings are presented within a training environment, if the CS is sufficiently salient, and if it is also the most informative stimulus

I am very grateful to John W. Moore and to H. C. Plotkin for their criticisms and comments on earlier drafts. present, then the CS should "overshadow" all other stimuli present, including background stimuli (Mackintosh, 1976). In this case, salience refers to all aspects of a stimulus which affect its conditionability other than its informational value, for example its intensity and modality, whereas informativeness refers exclusively to the capacity of a stimulus to act as a reliable predictor of a specified UCS.

A growing body of evidence shows that background stimuli can block conditioning to a CS (Dweck \& Wagner, 1970; Kremer, 1974; Tomie, 1976; Welker, Tomie, Davitt, \& Thomas, 1974). These studies have generally involved the pretreatment of background stimuli independently of the CS, either by pairing the training environment with unsignaled UCSs (e.g., Tomie, 1976) or by interpolating periods of exposure to the training environment alone during training (e.g., Dweck \& Wagner, 1970), the latter strategy reducing rather than increasing blocking. Blocking by background stimuli is then inferred from measures of the subjects' reactions to introduced or reintroduced CSs within the treated environment.

Less evidence is available regarding the capacity of a CS to overshadow background stimuli. A difficulty is that the overshadowing of background stimuli cannot be demonstrated adequately by measurements of the posttraining reactions of subjects to a nominal CS. A convincing demonstration requires the direct assessment of conditioning in terms of the back- 
ground stimuli themselves. Odling-Smee (1975a, 1975b) has reported two studies in which background stimuli were tested directly. In the first, groups of rats were locked in a box compartment for which they had previously demonstrated a preference. They were then exposed to noise CSs and to unavoidable shock UCSs. CS-UCS correlation was varied across groups by varying the $\mathrm{P}(\mathrm{US} / \mathrm{CS})$ and the $\mathrm{P}(\mathrm{US} /$ NoCS) contingencies. After training, all subjects were given a choice between alternative compartments in the absence of both the CS and the UCS. Subjects trained at high levels of CS-UCS correlation returned to the originally preferred compartment in spite of having received shock in it, but subjects trained at lower correlation levels did not. In the second study, the same general procedure was adopted but the interval between CS onset and UCS onset (i.e., the ISI) was varied across groups. Overshadowing again occurred but only over a limited range of ISI values. Both studies indicated that while a nominal CS can overshadow background stimuli, its capacity to do so is highly dependent upon its relationship with the UCS. Using the same paradigm as in these previous studies, the present study investigated some further effects of manipulating the CS-UCS relationship, with the aim of establishing precisely what constitutes the functional stimulus when a nominal CS overshadows background stimuli.

\section{EXPERIMENT 1}

The first experiment explored the role of the potentially informative temporal cues that are provided by an intermittent CS. It sought to find out whether a CS must provide temporal cues which are capable of signaling the precise moment of UCS onset before it can overshadow background stimuli, or whether overshadowing will still occur under conditions in which the CS is only capable of providing imprecise temporal cues, but in which a high level of CS-UCS correlation, and a high degree of CS-UCS contiguity, is maintained.

Typically, the only features of a nominal CS which can signal the moment of UCS onset with precision are CS onset and CS offset. The informativeness of both these events depends largely upon CS-UCS correlation and CS-UCS contiguity. However, in the present case, in order to separate out the effect of providing precise temporal cues from other conditioning variables, it was necessary to find ways of manipulating the informativeness of both the onset and offset of a CS which were as far as possible independent of CS-UCS correlation and CS-UCS contiguity. The strategy chosen used combinations of either a trace or a delay conditioning procedure with a CS either of fixed duration or of variable and unpredictable duration. The rationale behind this approach was as follows.
The trace procedure determined that CS offset always preceded the onset of the UCS by a constant temporal interval. Under the trace condition, therefore, CS offset became an informative event which potentially provided the subject with a precise temporal cue relative to the moment of UCS onset. The delay procedure, on the other hand, determined that CS offset always occurred after UCS onset. Under the delay condition, therefore, CS offset was uninformative, since it provided no predictive temporal cue relative to UCS onset. Similarly, the CS of fixed duration determined that $\mathrm{CS}$ onset invariably preceded UCS onset by a constant temporal interval. Under the fixed-CS condition, therefore, CS onset could serve as a precise temporal cue for the moment of UCS onset. Conversely, the CS of variable and unpredictable duration determined that there was no fixed interval between CS onset and UCS onset. Under the variable-CS condition, therefore, CS onset was uninformative, since it provided no precise temporal cue relative to UCS onset.

By combining these four conditions, both the number and the nature of the precise temporal cues given to any one group of subjects could be manipulated. Thus the combination of the trace procedure with the fixed-duration CS determined that both the onset and the offset of the CS could act as precise temporal cues relative to UCS onset. Alternatively, the combination of the delay procedure with the fixedduration CS preserved the potential informativeness of CS onset, but destroyed that of CS offset. The combination of the trace procedure with the variableduration CS destroyed the potential informativeness of CS onset, but preserved that of CS offset. Finally, the combination of the delay procedure with the variable-duration CS destroyed the potential informativeness of both CS onset and CS offset. None of these manipulations affected the level of CS-UCS correlation. This variable could be and was separately manipulated by running additional groups. Similarly, although the degree of temporal contiguity between the CS and UCS presentations was inevitably affected by the delay vs. trace manipulation, this factor could be minimized by using a CS of relatively long duration and a trace interval of relatively short duration.

\section{Method}

Subjects. The subjects were 70 experimentally naive male hooded rats (NIMR-f strain) obtained from the Medical Research Council Breeding Unit, London. Their weights at the start of the experiment varied between 300 and $350 \mathrm{~g}$. Throughout the experiment, all animals were maintained on an ad-lib food and water schedule. The animals were individually housed in cages partially affected by the natural diurnal light-dark cycle. The average daytime light intensity in the cages was $5.3 \mathrm{~cd} / \mathrm{m}^{2}$. The animals were run during daylight hours only, the intersession time for each individual subject being approximately $24 \mathrm{~h}$.

Apparatus. The apparatus consisted of a box structure divided into two unequal compartments, a larger white compartment 
( $40 \mathrm{~cm}$ wide, $40 \mathrm{~cm}$ long, $13 \mathrm{~cm}$ deep) and a smaller black compartment $(15 \mathrm{~cm}$ wide, $19 \mathrm{~cm}$ long, $13 \mathrm{~cm}$ deep). The walls and floor of the larger compartment were flat-white fiberboard. The compartment was fitted with a transparent Perspex lid. The walls and lid of the smaller compartment were a different material, flatblack hardboard. This compartment was fitted with an electrifiable grid floor. The two compartments were separated by a manually operated transparent Perspex sliding door. Ambient lighting conditions were arranged so that the average light intensity in the black compartment was $7.59 \mathrm{~cd} / \mathrm{m}^{2}$ and in the white compartment, $85.5 \mathrm{~cd} / \mathrm{m}_{2}$. The CS was a wide-frequency $74 \mathrm{~dB}$ (re $20 \mu \mathrm{N} / \mathrm{m}^{2}$ ) noise. The UCS was a footshock applied through the grid floor of the black compartment and delivered by a Grason-Stadler shock generator with nominal settings of $.5 \mathrm{sec}$ and $.5 \mathrm{~mA}$.

Procedure. Three variables were manipulated. The variables were correlated vs. uncorrelated presentations of CS and UCS; a trace vs. a delay-conditioning paradigm; and a CS of fixed duration vs. a CS of variable and unpredictable duration. Six experimental groups were run: Group TF, (trace, fixed CS); Group DF (delay, fixed CS); Group TV (trace, variable CS); Group DV (delay, variable CS); Group RF (random, fixed CS); and Group RV (random, variable CS). There were 10 subjects in each of Groups DF, TV, DV, and RF and 8 in both Groups TF and $\mathrm{RV}$.

For Groups TF, DF, TV, and DV the noise CS was always paired with the shock UCS. For groups RF and RV, the CS and UCS presentations were uncorrelated in a manner to be described. For the delay condition (Groups DF and DV), the last $.5 \mathrm{sec}$ of the CS overlapped with the presentation of the UCS regardless of the duration of the CS. For the trace condition (Groups TF and TV), a fixed trace interval of $3.0 \mathrm{sec}$ was interpolated between the offset of the CS and UCS onset, again regardless of CS duration. For the fixed-CS condition (Groups DF, TF, and RF), the duration of the CS was $42.0 \mathrm{sec}$. This value was selected even though it was known to exceed the optimal ISI (Odling-Smee, 1975b), because of the need to allow scope for adequate CS variability in the case of the variable-CS groups. For the variable-CS condition (Groups DV, TV, and RV), five different values of CS duration were given. These were $6.6,13.3,32.5,65.0$, and $98.0 \mathrm{sec}$. Variability was achieved by presenting each subject with each of these durations twice during the training session but with the sequence of presentations separately randomized for each individual subject. The mean CS duration for these groups was $43.1 \mathrm{sec}$. (The discrepancy between this value and the duration of the fixed CS was due to the imperfect calibration of one timer.)

Two control groups were also run. Group $\mathrm{Cl}$ received neither CSs nor UCSs during training. Group $\mathrm{C} 2$ received UCSs but not CSs. Six subjects were allocated to Group $\mathrm{Cl}$ and eight to Group C2.

The procedure was similar to that fully described in a previous study (Odling-Smee, 1975a). It comprised three phases. Phase 1 (Day 1) consisted of an initial compartment-preference test. The subject was placed in the white compartment of the apparatus for $300 \mathrm{sec}$. It was free to move throughout either or both compartments. The cumulative times spent in each compartment were recorded. Subjects which spent more than $120 \mathrm{sec}$ in the white compartment were rejected. The rejection rate was approximately $10 \%$. The remaining subjects were then allocated at random to groups.

Phase 2 (Day 2) consisted of the training stage. The subject was locked in the black compartment for a 30-min training session. During this period, all subjects from Groups DF, DV, TF, and TV received a total of $10 \mathrm{CS}$-UCS pairings. The CS-UCS pairs were presented at random intervals, the intervals being derived from a table of random numbers. There was, therefore, no regular intertrial interval. Randomization was limited by two superimposed restrictions. The minimum interval permitted between consecutive CS-UCS pairs was $15 \mathrm{sec}$. Also, no CS or UCS was ever permitted to occur during either the first minute or the last 2 min of a train- ing session. To eliminate possible artefacts due to sequence effects, similar to those reported by Benedict and Ayres (1972), a separate sequence of stimulus presentations was derived for each individual subject. Exactly the same training procedure was given to Groups RF and RV, except that for these two groups the $10 \mathrm{CSs}$ and 10 UCSs were presented as independently randomized events. In their case, the $15-\mathrm{sec}$ minimum interval rule applied only to like events. Unlike events were allowed to coincide. Thus it was possible for a UCS to occur by chance during an ongoing CS presentation. The two control groups were also treated in exactly the same way except that, for Group C1, no stimuli and, for Group C2, no CSs were ever presented.

Immediately after the training session, the door between the two compartments was raised, without the subject's being removed from the black compartment. A 300-sec "stabilization" period followed, during which the subject was again free to move throughout either compartment. The reason for inserting this period was that during pilot studies it was found that it reduced the freezing behavior demonstrated by subjects during the posttraining tests. Why this was so was not fully understood. At the end of the stabilization period, the subject was replaced in its home cage.

Phase 3 (Days 2 and 3 ) consisted of two posttraining preference tests. After $60 \mathrm{sec}$ in its home cage, the subject was replaced in the white compartment for a test period of $300 \mathrm{sec}$ (Test 1). A second test (Test 2) was given approximately $24 \mathrm{~h}$ later. The procedure for both of these tests was identical to that used for the initial pretraining preference test.

\section{Results and Discussion}

All the results are given in terms of "B scores," defined as the total number of seconds out of a maximum of $300 \mathrm{sec}$, spent by a subject with all four feet placed on the grid in the black compartment. A high B score indicates little or no reluctance to enter the black compartment. A low B score indicates considerable reluctance.

On the initial pretraining preference test, the differences among groups were not statistically significant. The highest mean score obtained from an individual group was 252.2 (Group DV); the lowest was 212.0 (Group C1).

The results of Tests 1 and 2 are shown in Figure 1. Since the B scores represented latency data with an arbitrary cut-off point of $300 \mathrm{sec}$ and were not normally distributed, they were analyzed by nonparametric tests, the Kruskal-Wallis and the MannWhitney. Significant differences were found among the six experimental groups on Test $1[\mathrm{H}(5)=12$, $\mathrm{p}<.05]$. This showed that the CS overshadowed the background stimuli at least partially under some conditions in spite of the fact that the ISI values tested were greater than the known optimal value (OdlingSmee, 1975b). Each experimental group was then individually compared with Group $\mathrm{C} 2$, the shocked control. It was found that, on Test 1, Group C2 avoided the black compartment significantly more than did Groups TF $(U=14 ; p<.032)$ and TV $(U=$ $19 ; \mathrm{p}<.05$ ), but it did not differ from any other groups. On Test 2, the B scores of Groups TF, DF, and TV all increased, but there were no changes in 

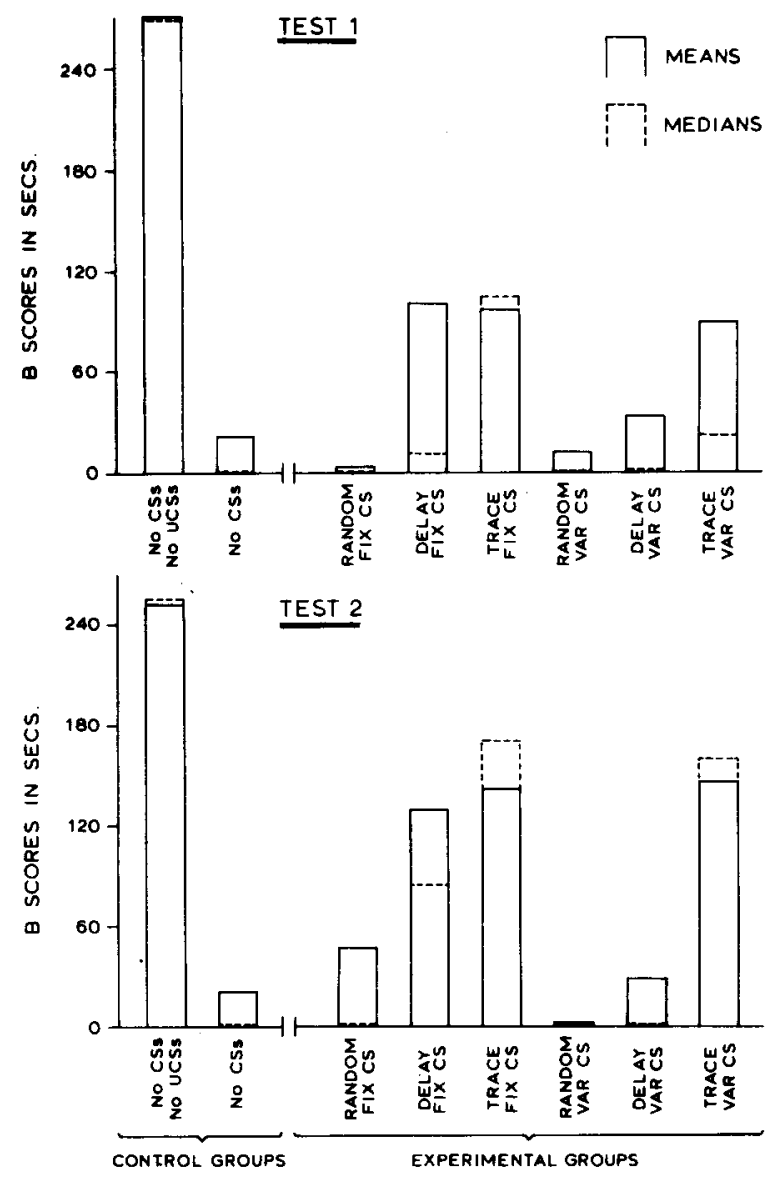

Figure 1. Experiment 1. Test 1 and Test 2 mean and median B scores for all groups. B scores refer to total time in seconds spent by subjects in the training environment (black compartment) after training.

the performances of any of the other groups. This meant that the difference between Group C2 and Group DF became significant on Test $2(U=19$; $\mathrm{p}<.05$ ). The reentry of Groups TF, DF, and TV into the black compartment after training showed that the noise CS developed some capacity to overshadow background stimuli under every condition in which it provided a precise temporal cue as to the moment of shock onset, regardless of whether this cue was provided by noise onset or noise offset, or both. Conversely, the failure of Groups DV, RF, and $\mathrm{RV}$ to reenter the black compartment on both tests showed that the CS failed to overshadow background stimuli under every other condition.

Individual group comparisons among the six experimental groups confirmed this finding. On Test 1 , Group DV avoided the black compartment significantly more than did Groups TF $(U=20 ; p<.05)$ and TV $(U=27 ; p<.05)$, and on Test 2 it also avoided the black compartment significantly more than did Group DF $(U=27 ; p<.05)$. It did not dif- fer from Groups RF and RV on either test. This showed that even though, for Group DV, both a high level of CS-UCS correlation and a high degree of CS-UCS contiguity were preserved, these factors were not enough in the absence of any precise temporal cues to permit the CS to overshadow the background stimuli. In fact, for Group DV, the CS must have provided imprecise temporal cues relative to UCS onset, and it must also have been the best predictor of the UCS available in the environment. Nevertheless, for Group DV, the CS was as ineffective a stimulus as it was for Groups RF and RV, the two groups in which the CS and UCS presentations were randomized.

Finally, no significant differences were found between Groups TF, DF, and TV on either test. This lack of difference indicated that the temporal cue provided by noise onset (Group DF) was approximately equivalent to the temporal cue provided by noise offset (Group TV), and that the provision of both cues (Group TF) as opposed to either cue (Groups DF and TV) did not significantly improve the capacity of the CS to overshadow background stimuli.

\section{EXPERIMENT 2}

The similarity of the results obtained from Groups DV, RF, and RV in Experiment 1 raised the question of whether CS-UCS correlation and CS-UCS contiguity affect the overshadowing of background stimuli other than by contributing towards the informativeness of the temporal cues provided by the onset and offset of a CS. Possibly the overshadowing of background stimuli depends exclusively on these temporal cues. If not, then what other factors, due either to CS-UCS correlation or to CS-UCS contiguity, must be satisfied? Experiment 2 set out to investigate one aspect of this question. Specifically, it aimed to dissociate a precise temporal cue, provided by the onset of a CS, from every other factor which might arise from CS-UCS contiguity. The relative contributions of the temporal cue presented in the absence of CS-UCS contiguity and of CS-UCS contiguity presented in the absence of any precise temporal cue could then be separately assessed.

The overall design was as follows: First, a "silent CS" was substituted for a "noise CS" for some groups. The silent CS consisted of an interval of silence which interrupted otherwise continuous noise. The noise CS was a conventional stimulus and consisted of an interval of noise which interrupted otherwise continuous silence. The purpose of this step was to check whether a precise temporal cue provided by the onset of a silent CS (i.e., noise offset) is as effective as a precise temporal cue provided by the onset of a noise CS (i.e., noise onset). A second 
step involved manipulating the configuration of background stimuli present in the environment during training and test sessions. For every group that was trained with the silent CS, a background noise was presented at all times during the training session except during the actual presentations of the silent CS itself. For half of the silent-CS groups, the same noise was also presented continuously during both the pretraining and test sessions, but for the other half of these groups silence was presented continuously during both the pretraining and test sessions. A consequence of this procedure was that for every silent-CS group, one component of the silent CS, namely its ongoing silence, was present during training when it was paired with the UCS. For half the silent-CS groups, however, this component was also presented throughout both the pretraining and test sessions, whereas for the other half of these groups it was never presented during either the pretraining or the test sessions. Conversely, for all the silent-CS groups, a second component of the silent CS, namely the precise temporal cue provided by CS onset (i.e., noise offset), was likewise present during training, when it was likwise paired with the UCS. But this component of the silent CS was never presented to any group at any other time. In this way, the ongoing silence of the silent CS was dissected from the CS and was added to the configuration of background stimuli already present in the subject's environment during pretraining and testing. The precise temporal cue provided by CS onset was thereby dissociated from the effects of pairing the ongoing silence of the silent CS either with the background stimuli during pretraining or with the UCS during training.

\section{Method}

Subjects. Subjects were 86 experimentally naive male hooded rats (NIMR-f strain) obtained from O.L.A.C., Bicester, England. Their weights at the start of the experiment varied between 280 and $350 \mathrm{~g}$. They were housed and maintained in exactly the same way as were subjects in Experiment 1.

Apparatus. Experiment 2 was carried out in a different laboratory from Experiment 1 , and there were several minor changes in the apparatus and procedure. The white compartment of the box structure became $44 \mathrm{~cm}$ wide, $43 \mathrm{~cm}$ long, $18 \mathrm{~cm}$ deep, and the black compartment became $15 \mathrm{~cm}$ wide, $18 \mathrm{~cm}$ long, $18 \mathrm{~cm}$ deep. The door separating the two compartments was changed to an opaque fiberboard door. Ambient lighting conditions were also altered to read $7.59 \mathrm{~cd} / \mathrm{m}^{2}$ in the black compartment and $153.9 \mathrm{~cd} / \mathrm{m}^{2}$ in the white compartment. The noise $\mathrm{CS}$ and all intervals referred to as "noise" consisted of an $89-\mathrm{dB}$ (re $20 \mu \mathrm{N} / \mathrm{m}^{2}$ ) pulse/sec click. It was delivered by a Campden Instruments click generator. The silent $\mathrm{CS}$ and all intervals referred to as "silent" actually consisted of a 42-dB (re $20 \mu \mathrm{N} / \mathrm{m}^{2}$ ) level of room noise. The UCS was a footshock delivered to the grid floor of the black compartment by a Grason-Stadler shock generator with nominal settings of $1.0 \mathrm{sec}$ and $.6 \mathrm{~mA}$.

Procedure. In general, the procedure was similar to that used in Experiment 1 except that a more favorable ISI was used and the amount of training was increased. Nine experimental groups were run with eight subjects per group. The groups were distributed across three different conditions, designated silence-noisesilence (SNS), noise-silence-noise (NSN), and silence-silence-silence (SSS). SNS referred to a condition in which subjects were tested in silence both before and after training but in which they received the noise CS during training whenever a CS was presented. NSN referred to a condition in which subjects were tested in noise both before and after training, but in which they received the silent CS during training whenever a CS was presented. SSS referred to a condition in which subjects were tested in silence both before and after training and in which they received the silent CS during training whenever a CS was presented.

Three groups were allocated to each of these conditions. In each case, the three groups were designated DF (delay, fixed CS), RF (random, fixed CS), and No CS. For the three DF groups, the CS was invariably paired with the shock UCS. The CS itself was fixed at $10 \mathrm{sec}$ regardless of which CS was presented. Also, a delay paradigm was used which determined that the last $1 \mathrm{sec}$ of the CS overlapped with the UCS presentation. The CS and UCS coterminated, making the ISI $9 \mathrm{sec}$. Each RF group was treated in exactly the same way as the DF group within its particular condition, except that the CSs and UCSs were presented independently. Similarly, each No-CS group received exactly the same treatment as the DF and RF groups within its particular condition except that for the No-CS groups only UCSs were presented.

Two control groups were also run with seven subjects per group. Group Cl was treated identically to Group DF in the SNS condition except that neither the noise CS nor the UCS was ever presented. Group C2 was treated identically to Group DF in the NSN condition except that neither the silent CS nor the UCS was ever presented.

The procedure was the same as for Experiment 1 with the following exceptions. Phase 1 (Day 1): All subjects were given a preliminary 20-min "familiarization period" in the apparatus 4-6 $\mathrm{h}$ prior to their initial pretraining compartmental preference test. During this period, the subjects were free to move throughout the entire apparatus. Phase 2 (Days 2-5): Four days of training were given. On each day, the subject was locked in the black compartment for a 30-min training session. During each session, the three DF groups each received either 10 noise CS-UCS or 10 silent CS-UCS pairings, whichever was appropriate. For the three RF groups, the same total number of stimuli were presented within each session, but they were separately programmed and randomized events, exactly as in Experiment 1. For the three No-CS groups, 10 UCSs only were delivered per session. Immediately after each of the first 3 days of training, the subject was removed from the black compartment and returned to its home cage until the following day. After the fourth and last training session, the door between the two compartments was raised without the subject's being removed from the apparatus. The subject was then given a 600 -sec stabilization period, during which it was free to move throughout both compartments. For the SNS and SSS conditions, this stabilization period always occurred in silence, but for the NSN condition, it always occurred in noise. The duration of the stabilization period was doubled over that used in Experiment 1 because pilot studies had shown that the altered training conditions in Experiment 2 increased freezing behavior during tests. The increased freezing could, however, be readily countered by increasing the stabilization period. Again, no reason can be offered for this finding.

\section{Results and Discussion}

On the initial pretraining preference test, the differences among groups were not statistically significant. The highest mean score obtained from an individual group was 268.2 (Group RF, condition NSN); the lowest was 232.0 (Group C1). 

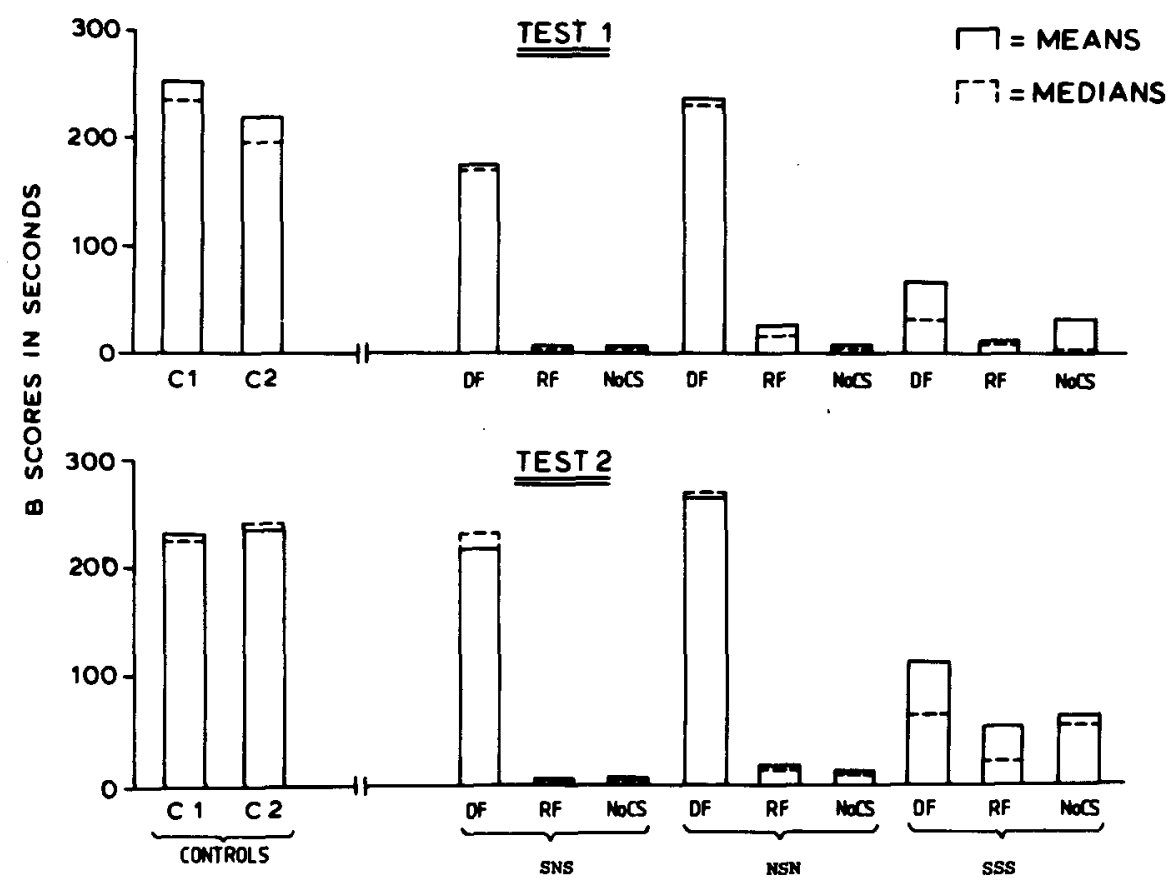

Figure 2. Experiment 2. Test 1 and Test 2 mean and median $B$ scores for all groups. SNS refers to the silence-noise-silence condition. NSN refers to the noise-silence-noise condition. SSS refers to the silence-silence-silence condition.

Test 1 and 2 results are shown in Figure 2. The two control groups did not differ from each other on either test, indicating that merely adding a continuous noise to the experimental situation did not affect the subjects' basic compartmental preferences.

A significant difference was found among the nine experimental groups on Test $1[\mathrm{H}(8)=38.4$, $\mathrm{p}<.001]$. Between-groups comparisons showed that within both the SNS and the NSN conditions there was no overlap between the scores obtained by the DF groups, on the one hand, and by the RF and No-CS groups, on the other. Under both of these conditions, the DF groups showed no reluctance to reenter the black compartment after training, whereas the RF and No-CS groups showed almost complete avoidance. The close similarity between the two DF groups also indicated that the silent CS must have been as effective a stimulus as the noise CS. It implied that ongoing noise in the NSN condition was equivalent in every respect to ongoing silence in the SNS condition. Thus, for the DF group in the NSN condition, ongoing noise must have acquired the status of just another background stimulus and, as such, it must have been overshadowed by the silent CS. These results confirmed the Experiment 1 finding that a temporal cue provided by noise onset can be equivalent to a temporal cue provided by noise offset. They also supported data obtained from earlier studies in which learning was measured rela- tive to a nominal CS rather than relative to background stimuli and which demonstrated the equivalence of CS onset and CS offset (Champion, 1962; Logan \& Wagner, 1962; Myers, 1960). Within the third SSS condition, it was found that animals from Group DF reentered the black compartment significantly more than did those from the No-CS group $(U=13 ; p<.025)$, but here Group DF did not differ from Group RF $(U=22 ; p<.164)$. This indicated that the temporal cue provided by the silent CS must have had a significant but marginal effect.

A comparison across the NSN and SSS conditions showed that Group DF in the NSN condition differed significantly from Group DF in the SSS condition $(U=5 ; p<.002)$. Since these two groups were treated identically during training, the difference between them can only have been due to their different treatments either prior to or after training.

One possibility is that, in the SSS condition, Group DF never conditioned well to the silent CS because latent inhibition developed to silence during the initial pretraining phase (Lubow, 1973). If so, then the capacity of the silent CS to overshadow background stimuli could have been reduced. The only way to rule out this possibility would be to run an extra NSS condition in which animals would be exposed to noise during pretraining but would thereafter be treated in the same way as animals in the present SSS condition. There are, however, grounds 
for supposing that latent inhibition cannot have made a major contribution to the present results. If a significant amount of latent inhibition had developed to silence during pretraining, it should have done so in the case of every group that was exposed to silence during this period. It ought, therefore, to have had some effect on the results of several groups. In particular, Groups RF and No CS in the SNS condition might have revealed signs of latent inhibition by showing only partial rather than complete avoidance of the black compartment after training. But they did not.

A more probable explanation of the difference between these two DF groups lies in their posttraining treatments. For Group DF in the NSN condition, all the features of the silent CS which had previously been paired with the UCS were absent during both the stabilization period and the tests. For Group DF in the SSS condition, on the other hand, one component of the silent CS, namely the temporal cue provided by CS onset, was absent, but another component, namely ongoing silence, was still present. If ongoing silence had previously acquired an association with the UCS as a function of its contiguity with the UCS during training, then its continuing presence during tests could account for the depressed B scores returned by this group.

Some support for this view comes from the RF and No-CS groups. In the SSS condition, Groups RF and No CS tended to avoid the black compartment during tests less than did the equivalent groups in both the other two conditions. This is consistent with the fact that for Groups RF and No CS in the SSS condition, but not for the same groups in either of the other two conditions, one background stimulus element, namely ongoing noise, was present during training but not present during tests. The subtraction of this element from the background could therefore have made the black compartment relatively less aversive.

Regardless of which of these two explanations account for the difference between the DF groups in the NSN and SSS conditions, it is evident that the observed difference cannot be accounted for exclusively in terms of the temporal cue that was provided by CS onset during training. Either the contiguity of ongoing silence with background stimuli during pretraining or the contiguity of ongoing silence with the UCS during training must have played some part in these results.

There is one other complication. For the DF group in the SSS condition, ongoing silence was present in both compartments of the apparatus during tests. It might therefore have been expected that the posttraining associative strength of silence in the white compartment should have cancelled out the posttraining associative strength of silence in the black compartment, leaving the subjects' initial preference for the black compartment intact. The results demonstrated that subjects did, in fact, discriminate between silence in compound with the black compartment and silence in compound with the white compartment. This raises the possibility that the relationship between an overshadowing CS and background stimuli may be affected by configural conditioning factors (Rescorla, 1972, 1973). It may not always be possible to predict a subject's reactions to stimulus compounds, inclusive of background stimuli, solely in terms of the summation of their reactions to each separate component within the compounds. Unfortunately, the evidence provided by the present experiment was too scanty to settle this issue.

\section{GENERAL DISCUSSION}

The present experiments established two points. Experiment 1 showed that close CS-UCS contiguity coupled to a high level of CS-UCS correlation is not sufficient to insure that a nominal CS will develop a capacity to overshadow background stimuli. An effective CS must provide at least one precise temporal cue relative to the moment of UCS onset as a function of its own intermittence. Conversely, Experiment 2 showed that the provision of a precise temporal cue by a CS is also not sufficient to insure that the CS will overshadow background stimuli. Other factors arising out of the contiguity of an ongoing CS with the UCS presentations are potent.

Both experiments nevertheless demonstrated that a nominal CS can overshadow background stimuli if and when the CS-UCS relationship meets certain minimal requirements. Specifically, if a nominal CS (1) is highly correlated with a UCS, (2) provides at least one precise temporal cue relative to the moment of UCS onset, and (3) is presented in close contiguity with the UCS, then it will overshadow background stimuli. This finding complements those previously reported by Tomie (1976) and Welker, Tomie, Davitt, and Thomas (1974) which showed that background stimuli which have already acquired associative strength are able to compete successfully with a subsequently introduced $\mathrm{CS}$ by blocking it. The present study, in conjunction with others that have used the the same paradigm (Odling-Smee, 1975a, 1975b), shows that a nominal CS can likewise develop a reciprocal capacity to compete with background stimuli by overshadowing them.

One question which arises is whether the same minimal requirements apply to all Pavlovian conditioning situations, or whether they only apply to the overshadowing of background stimuli. In particular, must a CS always provide at least one precise temporal cue relative to UCS onset before learning will occur? An implication of the general correspondence 
between learning in the present situation and learning in the conditioned emotional response (CER) situation (Odling-Smee, 1975b) is that the answer may possibly be yes. However, the question itself has hitherto received insufficient attention to permit a definite answer. In this respect, curiosity has not been encouraged by the fact that at least one of the major contemporary models of Pavlovian conditioning (Rescorla \& Wagner, 1972) does not yet reference temporal parameters.

The present experiments do support the view that Pavlovian conditioning is always a function of a competition for associative strength between all the potentially conditionable stimuli present in a subject's environment (Mackintosh, 1975; McHose \& Moore, 1976; Rescorla \& Wagner, 1972; Sutherland \& Mackintosh, 1971). In particular, the results largely agree with Mackintosh's (1975) statement that overshadowing occurs either because animals learn to ignore stimuli that predict the outcome of a trial less well than other available stimuli or because they fail to increase their attention to stimuli which are not the most reliable predictors available. The fact that in Experiment 1 overshadowing did not occur when a nominal CS (which must have been the best predictor of the UCS available) was presented until that CS provided at least one precise temporal cue merely indicates that Mackintosh's (1975) statement, like Rescorla and Wagner's (1972) model, is probably still incomplete.

Finally, the results are consistent with Lubow, Rifkin, and Alek's (1976) hypothesis that learning centers around a figure-ground discrimination task. According to Lubow et al., the resolution of the figure-ground task is a function of a variable which they refer to as "contrast." Contrast is primarily defined in terms of stimulus novelty and stimulus salience, but it could equally well include the informativeness of stimuli. An implication of their theory is that the initial status of a CS is likely to resemble that of background stimuli, but that as a function of training, and therefore as a function of increasing contrast, the CS may develop a special status, and in doing so gain control over the subject's behavior. One advantage attaches to Lubow et al's hypothesis. The theories of Rescorla and Wagner, and of Sutherland and Mackintosh, model the competitive relationship between a CS and background stimuli, but they are not forthcoming as to why such a relationship should exist. However, Lubow, et al. do potentially provide an explanation. If Pavlovian conditioning consists of figure-ground discrimination, then the discrimination of the CS as part of the "figure" rather than as part of the "background" must involve the parallel discrimination of all other stimuli present in the environment as part of the "background" rather than as part of the "figure." Hence, during training, and particularly during the early stages of training, there will be a competition for control over the subject's behavior between all the stimuli present in the environment. In this light, the reciprocal phenomena of blocking and overshadowing assume an increased importance. Rather than seeming to be Pavlovian epiphenomena, blocking and overshadowing begin to appear as two principal processes by means of which the figure-ground discrimination task, possibly central to Pavlovian conditioning and possibly central to other learning paradigms as well, is resolved.

\section{REFERENCES}

Benedict, J. O., \& AYres, J. J. B. Factors affecting conditioning in the truly random control procedure in the rat. Journal of Comparative and Physiological Psychology, 1972, 78, 323-330.

Champion, R. A. Stimulus-intensity effects in response evocation. Psychological Review, 1962, 69, 428-449.

DWECK, C. S., \& WAGNER, A. R. Situational cues and correlation between CS and US as determinants of the conditioned emotional response. Psychonomic Science, 1970, 18, 145-147.

Kamin, L. J. Predictability, surprise, attention and conditioning. In B. Campbell \& R. Church (Eds.), Punishment and aversive behavior. New York: Appleton-Century-Crofts, 1969.

KREMER, E. F. The truly random control procedure: Conditioning to the static cues. Jourmal of Comparative and Physiological Psychology, 1974, 86, 700-707.

LogAN, F. A., \& WAGNeR, A. R. Supplementary report: Direction of change in CS in eyelid conditioning. Journal of Experimental Psychology, 1962, 64, 325-326.

Lubow, R. E. Latent inhibition. Psychological Bulletin, 1973, 79, 398-407.

Lubow, R. E., Rifkin, B., \& Alek, M. The context effect: The relationship between stimulus pre-exposure and environmental pre-exposure determines subsequent learning. Journal of Experimental Psychology: Animal Behavior Processes, 1976, 1, 38-47.

Mackintosh, N. J. A theory of attention: Variations in the associability of stimuli with reinforcement. Psychological Review, 1975, 82, 276.298.

Mackintosh, N. J. Overshadowing and stimulus intensity. Animal Learning and Behavior, 1976, 4, 186-192.

McHose, J. H., \& Moore, J. N. Expectancy, salience, and habit: A noncontextual interpretation of the effects of changes in the conditions of reinforcement on simple instrumental responses. Psychological Review, 1976, 83, 292-307.

MyERS, A. K. Onset vs. termination of stimulus energy as the CS in avoidance conditioning and pseudoconditioning. Journal of Comparative and Physiological Psychology, 1960, 53, 72-78.

ODLing-SmeE, F. J. The role of background stimuli during Pavlovian conditioning. Quarterly Journal of Experimental Psychology, 1975, 27, 201-209. (a)

ODLING-SMEE, F. J. Background stimuli and the inter-stimulus interval during Pavlovian conditioning. Quarterly Joumal of Experimental Psychology, 1975, 27, 387-392. (b)

Rescorla, R. A. Configural conditioning in discrete trial bar pressing. Journal of Comparative and Physiological Psychology, 1972, 79, 307-317.

Rescorta, R. A. Evidence for "unique stimulus" account of configural conditioning. Journal of Comparative and Physiological Psychology, 1973, 85, 331-338.

Rescorla, R. A., \& WAgner, A. R. A theory of Pavlovian conditioning: Variations in the effectiveness of reinforcement 
and nonreinforcement. In A. H. Black \& W. F. Prokasy (Eds.), Classical conditioning II. New York: Appleton-CenturyCrofts, 1972.

Sutherland, N. S., \& Mackintosh, N. J. Mechanisms of animal discrimination learning. New York: Academic Press, 1971.

TomIE, A. Interference with autoshaping by prior context conditioning. Journal of Experimental Psychology: Animal Behavior Processes, 1976, 2, 323-334.
Welker, R. L., Tomie, A., Davitt, G. A., \& Thomas, D. R. Contextual stimulus control over operant responding in pigeons. Journal of Comparative and Physiological Psychology, 1974, 86, 549-562.

\author{
(Received for publication June 1.1977; \\ revision accepted October $7,1977$.
}

\section{ERRATUM to Wheatley, Welker, and Miles}

In the article, "Acquisition of Barpressing in Rats Following Experience with Response-Independent Food," by K. L. Wheatley, R. L. Welker, and R. C. Miles (Animal Learning \& Behavior, 1977, 5, No. 3, 236-242), an error appears on page 239. The last sentence of the first paragraph of the left-hand column reads:

Newman-Keuls comparisons regarding the groups indicated that the response-contingent subjects completed the 50 barpresses faster than the controls which were in turn faster than the response-independent subjects (all post hoc comparisons were computed at .01 alpha levels).
It should read as follows:

Newman-Keuls comparisons regarding the groups indicated that the response-contingent subjects completed the 50 barpresses faster than the controls, which were in turn faster than the response-independent subjects, although only the difference between the responsecontingent and response-independent subjects was statistically significant $(p<.05)$.

(Editor's note: The error, which is an important one in terms of the conclusions drawn by the authors, was pointed out by William W. Beatty and William S. Maki, Jr., of North Dakota State University.) 